\title{
A plasmonic 'antenna-in-box' platform for enhanced single-molecule analysis at micromolar concentrations
}

\author{
Deep Punj ${ }^{1}$, Mathieu Mivelle ${ }^{2}$, Satish Babu Moparthi ${ }^{1}$, Thomas S. van Zanten ${ }^{2}$, \\ Hervé Rigneault ${ }^{1}$, Niek F. van Hulst ${ }^{2,3}$, María F. García-Parajó ${ }^{2,3}$, and Jérôme Wenger ${ }^{1 *}$ \\ ${ }^{1}$ CNRS, Aix-Marseille Université, Ecole Centrale Marseille, Institut Fresnel, Campus de St Jérôme, \\ 13397 Marseille, France \\ 2 ICFO-Institut de Ciences Fotoniques, Mediterranean Technology Park, 08860 Castelldefels, Spain \\ ${ }^{3}$ ICREA-Institució Catalana de Recerca i Estudis Avançats, Barcelona, 08010, Spain \\ * Corresponding author: jerome.wenger@fresnel.fr
}

Single molecule fluorescence techniques [1-3] are key for several applications including DNA sequencing $[4,5]$, molecular and cell biology $[6,7]$, and early diagnosis [8]. Unfortunately, observation of single molecules by diffraction-limited optics is restricted to detection volumes in the femtolitre range and imperatively requires pico- or nanomolar concentrations, far below the micromolar range where most biological reactions occur [2]. This limitation can be overcome using plasmonic nanostructures, and confinement of light down to nanoscale volumes has been reported [9-13]. While these nanoantennas enhance fluorescence brightness [14-20], large background signals [20-22] and/or unspecific binding to the metallic surface [23-25] has hampered the detection of individual fluorescent molecules in solution at high concentrations. Here we introduce a novel "antenna-in-box" platform that is based on a gap-antenna inside a nanoaperture. This design combines fluorescent signal enhancement and background screening, offering high single molecule sensitivity (fluorescence enhancement up to 1100 folds and microsecond transit time) at micromolar sample concentrations and zeptolitre-range detection volumes. The antennain-box device can be optimized for single molecule fluorescence studies at physiologicallyrelevant concentrations, as we demonstrate using various biomolecules.

Our antenna-in-box design is shown in Figure 1a and b. The rationale behind our design is that in any nanoantenna experiment on molecules in solution, the observed fluorescence signal is a sum of two contributions: the enhanced fluorescence from the few molecules in the nanoantenna gap region (hot 
spot) and a fluorescence background from several thousands of molecules within the diffraction-limited confocal volume. Thus the different components of our antenna-in-box have complementary roles: a central gap-antenna creates the hot spot for enhancement (Fig. 1c), while a surrounding nanoaperture screens the background by preventing direct excitation of molecules diffusing away from the central gap region. This configuration maximizes the signal-to-background discrimination by singling out the fluorescence signal from the hot spot while several thousands of non-excited molecules are present in the confocal volume.

The antenna-in-box is directly milled in a $50 \mathrm{~nm}$ thick gold film on a glass coverslip by focused ion beam (FIB). Here, the half-sphere diameter is $76 \mathrm{~nm}$ with gap sizes varying from 12 to $40 \mathrm{~nm}$, and the nanoaperture dimensions are 290x100 nm² (Fig. 1b). The FIB fabrication provides well-controlled sets of nanoantennas at defined locations (Fig. 1d). Moreover, by scaling up the FIB prototypes by nanoimprint lithography and other replication techniques, the fabrication of thousands of such antennas can be envisioned on a microscope coverslip.

Beyond local enhancement and screening by the antenna-in-box, the molecular photodynamics can be exploited to further improve the contrast between the hot spot enhanced emission and the background fluorescence. Fluorescent emitters with low quantum yield exhibit much higher enhancement factors than emitters with high quantum yield, because their low quantum yield takes larger benefit from nanoantenna enhancement $[16,17]$. Here we use this concept to gain extra contrast: we introduce a chemical quencher (methyl viologen) to lower the quantum efficiency of Alexa Fluor 647 (A647) molecules. With quencher concentrations of $200 \mathrm{mM}$, the quantum yield of A647 reduces from $30 \%$ to $8 \%$ (see Supporting Information).

For direct observation of single molecule diffusion events, the nanoantennas are covered by a solution containing the fluorescent dyes and the chemical quencher. The molecules constantly diffuse in a Brownian fashion around the nanoantenna, thus the configuration is virtually not limited by photobleaching. The fluorescence signal $F$ is analyzed by fluorescence correlation spectroscopy (FCS) to quantify the detection volume and fluorescence brightness per emitter $[17,26]$. FCS computes the temporal correlation of the fluorescence signal $G(\tau)=\langle F(t) . F(t+\tau)\rangle /\langle F(t)\rangle^{2}$, where $\tau$ is the delay (lag) time, and \langle\rangle indicates time averaging. For a homogeneous sample with a single fluorescent species, the amplitude of the correlation function $G$ scales with the inverse of the average number of molecules in the detection volume $N$, and provides access to the fluorescence brightness per molecule $Q=\langle F\rangle / N$. In the case of the nanoantenna, the FCS curves are analyzed with a model considering two species with different brightness (see Supporting Information Section 3): $N^{*}$ molecules in the hot spot volume with brightness $Q^{*}$, and $N_{0}$ background molecules with brightness $Q_{0}$ diffusing away from the hot spot. An important feature of FCS is that the different fluorescent species contribute to the amplitude of $G$ in proportion to the square of their fluorescence brightness [3]. Hence, a large fluorescence enhancement in the hot spot improves the signal-to-background contrast in FCS by a 
quadratic manner.

Confocal experiments at $15 \mu \mathrm{M}$ concentration collect a large fluorescence signal with reduced temporal fluctuations (Fig. 2a,b). The FCS analysis measures an average number of $N_{\text {sol }}=4630$ molecules, corresponding to the $15 \mu \mathrm{M}$ concentration and the $0.5 \mathrm{fL}$ detection volume calibrated independently. The brightness per molecule in the presence of the quencher is $Q_{\text {sol }}=760 / 4630=$ $0.17 \mathrm{kHz}$. The validity of the experimental data in Fig. 2b has been carefully checked by performing successive supplementary dilutions. In contrast to the confocal case, the fluorescence time traces for nanoantennas (Fig. 2c) have lower intensity levels but larger relative fluctuations. FCS data display remarkably higher correlation amplitudes, shorter diffusion times and excellent signal-to-noise characteristics (Fig. 2d). Such features can only be obtained by monitoring a small number of molecules with high apparent brightness from a small detection volume. In the case of excitation polarisation parallel to the dimer axis, we obtain an average number of $N^{*}=0.96$ molecules in the hot spot with brightness $Q^{*}=128.7 \mathrm{kHz}$, which corresponds to a fluorescence enhancement of $Q^{*} / Q_{\text {sol }}=758$. The detection volume reduction is defined as the ratio of the number of molecules detected in the confocal volume to the number of molecules detected in the antenna hot spot. Here, the volume reduction is thus $N_{\text {sol }} / N^{*}=4820$, which corresponds to a hot spot volume of $104 \mathrm{zL}\left(1 \mathrm{zL}=10^{-21} \mathrm{~L}\right)$. The influence of the excitation laser polarisation (Fig. 2c,d) confirms that the fluorescence signal stems from the nanoantenna hot spot. These experimental results stand in full agreement with the values inferred from the numerical simulations (a discussion about the different decay rates is given in Section 10 of Supporting Information ).

Nanoantennas have excellent temporal resolutions. Normalized FCS curves for nanoantennas with parallel and perpendicular polarisations are shown in Fig. 2e in comparison to the confocal reference. In case of parallel excitation polarisation, the average residence time for molecules in the hot spot amounts to only $2.1 \mu \mathrm{s}$, nearly two orders of magnitude shorter than the confocal reference. This significant reduction in time scales highlights the distinction between molecules in the hot spot and molecules away in the confocal volume. Moreover, it indicates that in our experimental conditions, molecular adhesion remains minimal (see Supporting Information Section 7).

The antenna-in-box transforms a poor-quantum-yield dye into a very bright emitter: we detect count rates above $300 \mathrm{kHz}$ per molecule with the nanoantenna (Fig. 2f), while for the confocal setup A647 fluorescence saturates to values below $1 \mathrm{kHz}$ with methyl viologen, or $25 \mathrm{kHz}$ without quencher [17]. Importantly, the count rates with the nanoantenna significantly exceed those obtained with a high-quantum-yield emitter in a confocal microscope.

Local field enhancement critically depends on the antenna gap size. Figure $3 a$ and b show scatter plots of the fluorescence enhancement as a function of the volume reduction and gap size for 59 nanoantennas with gap sizes ranging from 12 to $40 \mathrm{~nm}$. Clear correlation between fluorescence enhancement and volume reduction consistent with field localization in the gap region is observed. The 
nanoantennas with smallest gap sizes yield the highest fluorescence enhancement and volume reduction, up to 1100x enhancement factor and 8550x volume reduction compared to confocal excitation. The effective detection volume measured with FCS is plotted in Fig. 3c as a function of gap size. The best confinement achieved amounts to a volume down to $58 \mathrm{zL}$ for a gap size of $12 \mathrm{~nm}$. Remarkably, this detection volume is four orders of magnitude smaller than the diffraction limit. Extensive comparison between the antenna-in-box, antenna alone and box alone are presented in the Supporting Information Section 4.

The antenna-in-box platform can be successfully applied to a broad range of biochemical applications. We show in the Supporting Information Section 5 that our design is able to accurately quantify the number of detected molecules in the concentration regime exceeding $20 \mu \mathrm{M}$, and provide single molecule detection at relevant physiological concentrations. Here, we demonstrate the applicability of the antenna-in-box to investigate various biomolecules at 10 micromolar concentrations (Figure 4). We consider the cellular protein Annexin 5b labeled with Cyanine5 fluorescent dye, double stranded DNA constructs of 11, 40 and 51 base pairs (bp) labeled with Atto647N, and the cell wall surface protein Protein A labeled with Alexa Fluor 647. Annexins, DNA and Protein A are widely used in biological research and pharmaceutical applications at industrial scale. The choice of fluorescent labels (Alexa 647, Cyanine5 and Atto647N) covers the most commonly used probes for the red spectral region. The samples also include the use of the restriction enzyme PstI to cleave the DNA constructs into shorter strands (see Methods). Figure 4a compares the correlation functions obtained with the different molecular samples on an antenna-in-box of $15 \mathrm{~nm}$ gap size. A gradual shift of the correlation data to longer times is observed as the molar mass of the sample is increased. Figure $4 \mathrm{~b}$ plots the average residence time in the nanoantenna volume versus the molecular hydrodynamic radius (calibrated from confocal measurements, see Supporting Information Section 6). For the different samples tested, the average residence time in the nanoantenna is found to increase proportionally to the hydrodynamic radius. For proteins larger than the antenna gap size, exclusion effects and significant deviations from the linear trend are expected. The observed linear dependence also indicates that our data are not compromised by photobleaching, local heating or surface binding (see Supporting Information Section 7 for the use of a surfactant to avoid molecular binding). The antenna-in-box can thus be readily used to determine relative diffusion coefficients at high physiologically relevant concentrations, and to distinguish between different fluorescent species based on their diffusion properties. We note that these studies were performed in the absence of the quencher to avoid potential chemical reactions with the protein (see Supporting Information Section 14 about detection without chemical quencher).

Using the dominant fluorescence emission from the nanoantenna gap region together with background screening afforded by the surrounding box, we isolated detection volumes down to $58 \mathrm{zL}$, accompanied by a 1100-fold fluorescence enhancement. The antenna-in-box concept represents an optically efficient, and robust platform for performing a wide variety of biochemical studies with sin- 
gle molecule resolution at physiologically-relevant high concentrations. This preludes a new class of nanoscale biomolecular studies to investigate enzymatic reactions $[4,5]$, and nanoscale composition of live cell membranes $[6,7]$.

\section{Methods}

\section{Sample fabrication}

Nanoantennas are milled by focused ion beam (Zeiss Auriga 60 FIB-SEM, $1 \mathrm{~nm}$ resolution GEMINI SEM, equipped with Orsay Optics $2.5 \mathrm{~nm}$ resolution Cobra ion column) on $50 \mathrm{~nm}$ thick gold films deposited by thermal evaporation (Oerlikon Leybold Univex 350). Adhesion between the gold film and the glass coverslip substrate is ensured by a $3 \mathrm{~nm}$ thick titanium layer deposited by e-beam evaporation.

\section{Chemicals}

We perform experiments on Alexa Fluor 647 fluorescent molecules (A647, Invitrogen, Carlsbad, CA) at micromolar concentrations in a water-based phosphate buffered saline (PBS) solution, with $200 \mathrm{mM}$ of methyl viologen (1,1'-Dimethyl-4,4'-bipyridinium dichloride, Sigma-Aldrich). This concentration of methyl viologen quenches the A647 quantum yield from 30\% down to 8\% (see Stern-Volmer analysis in the Supporting Information Section 13). Double stranded DNA constructs (51 base pairs) labeled with Atto647N at varying distances from the restriction site (CTGCAG) were purchased from IBA GmBH, Germany. Upon PstI enzyme(Invitrogen) digestion sequences 1 and 2 yield 11 base pairs and 40 base pairs dsDNA labeled constructs respectively. PstI cleavage reaction on $1 \mu \mathrm{g}$ dsDNA was carried out at $37^{\circ} \mathrm{C}$ in a bufferH (Invitrogen) for 1 hour, followed by inactivation of enzyme at $65^{\circ} \mathrm{C}$ for 20 minutes. Sequence 1: 5'CGCACTGAACAGCATATGACACGCGATAGGCTATCCTGCA $\downarrow$ GTACGCTCAGG 3'. Sequence2: 5'CGCACTGAACAGCATATGACACGCGATAGGCTATCCTGCA $\downarrow$ GTACGCTCAGG 3'. The Protein A labeled with Alexa Fluor 647 was used as purchased from Invitrogen, and diluted in PBS buffer. A polysorbate surfactant (Tween 20, Sigma-Aldrich) was used at $1 \%$ concentration to avoid protein adsorption to the metal surfaces (see the Supporting Information Section 7 for a discussion about the influence of the surfactant).

\section{Experimental setup}

The experimental set-up is based on a confocal inverted microscope with a Zeiss C-Apochromat 40x 1.2NA water-immersion objective. For FCS measurements, the excitation source is a CW He-Ne laser operating at $633 \mathrm{~nm}$. Accurate positioning of the nanoantenna at the laser focus spot is obtained with a multi-axis piezoelectric stage (Polytech PI P-517.3CD). A dichroic mirror (Omega Filters 650DRLP) 
and a long pass filter (Omega Filters 640AELP) separate the fluorescence light from the epi-reflected laser and elastically scattered light. A $30 \mu \mathrm{m}$ confocal pinhole defines a detection volume calibrated to $0.5 \mathrm{fL}$. After the confocal pinhole, the detection is performed by two avalanche photodiodes (Micro Photon Devices by PicoQuant MPD-5CTC) with $670 \pm 20 \mathrm{~nm}$ fluorescence bandpass filters. The fluorescence intensity temporal fluctuations are analyzed with a hardware correlator (Flex02-12D/C correlator.com, bridgewater NJ with 12.5 ns minimum channel width). Each individual FCS measurement is obtained by averaging 10 runs of $5 \mathrm{~s}$ duration for the nanoantennas and 50 runs of $5 \mathrm{~s}$ for the confocal reference.

\section{Numerical simulations}

Three-dimensional numerical modelling on nanoantennas is based on the finite-difference time-domain FDTD method using Rsoft Fullwave version 6.0. The model considers a computational space of $0.36 \times 0.2 \times 0.1 \mu \mathrm{m}^{3}$ with $0.75 \mathrm{~nm}$ mesh size and perfect matched layers boundary conditions on all faces. The gold antenna (refractive index $0.183+2.974 \mathrm{i}$ ) is on a glass substrate (refractive index 1.52), the upper medium is water. The particle constituting the antenna is treated as a half-sphere of diameter $76 \mathrm{~nm}$, the gap size is $15 \mathrm{~nm}$, the aperture dimensions are $290 \times 100 \mathrm{~nm}^{2}$ and the gold film thickness is $50 \mathrm{~nm}$. Excitation at $633 \mathrm{~nm}$ is launched incoming from the glass side. Electromagnetic intensity is measured and averaged in the gap region over the plane $10 \mathrm{~nm}$ inside the antenna from the glass interface.

\section{References}

[1] Lu, H. P., Xun, L. \& Xie, X. S. Single-Molecule Enzymatic Dynamics. Science 282, 1877-1882 (1998).

[2] Craighead, H. G. Future lab-on-a-chip technologies for interrogating individual molecules. Nature 442, 387-393 (2006).

[3] Zander, C., Enderlein J. \& Keller, R. A. Single-Molecule Detection in Solution - Methods and Applications, VCH-Wiley, Berlin/New York, 2002.

[4] Eid, J., Fehr, A., Gray, J., Luong, K., Lyle, J., Otto, G., Peluso, P., Rank, D., Baybayan, P., Bettman, B., Bibillo, A., Bjornson, K., Chaudhuri, B., Christians, F., Cicero, R., Clark, S., Dalal, R., Dewinter, A., Dixon, J., Foquet, M., Gaertner, A., Hardenbol, P., Heiner, C., Hester, K., Holden, D., Kearns, G., Kong, X. X., Kuse, R., Lacroix, Y., Lin, S., Lundquist, P., Ma, C. C., Marks, P., Maxham, M., Murphy, D., Park, I., Pham, T., Phillips, M., Roy, J., Sebra, R., Shen, G., Sorenson, J., Tomaney, A., Travers, K., Trulson, M., Vieceli, J., Wegener, J., Wu, D., Yang, 
A., Zaccarin, D., Zhao, P., Zhong, F., Korlach, J. \& Turner, S. Real-Time DNA Sequencing from Single Polymerase Molecules. Science 323, 133-138 (2009).

[5] Uemura, S., Aitken, C. E., Korlach, J., Flusberg, B. A., Turner, s. W. \& Puglisi, J. D. Real-time tRNA transit on single translating ribosomes at codon resolution, Nature 464, 1012-1017(2010).

[6] Bacia, K., Kim, S. A. \& Schwille, P. Fluorescence cross-correlation spectroscopy in living cells. Nature Meth. 3, 83-89 (2006).

[7] Eggeling, C., Ringemann, C., Medda, R., Schwarzmann, G., Sandhoff, K., Polyakova, S., Belov, V. N., Hein, B., von Middendorff, C., Schönle, A. \& Hell, S. W. Direct observation of the nanoscale dynamics of membrane lipids in a living cell. Nature 457, 1159-1162 (2009).

[8] Pitschke, M., Prior, R., Haupt, M. \& Riesner, D. Detection of single amyloid $\beta$-protein aggregates in the cerebrospinal fluid of Alzheimer's patients by fluorescence correlation spectroscopy. Nature Med. 4, 832-834 (1998).

[9] Levene, M. J., Korlach, J., Turner, S. W., Foquet, M., Craighead, H. G. \& Webb, W. W. Zero-mode waveguides for single-molecule analysis at high concentrations. Science 299, 682-686 (2003).

[10] Novotny, L. \& van Hulst, N. Antennas for light. Nature Photon. 5, 83-90 (2011).

[11] Biagioni, P., Huang, J. S. \& Hecht, B. Nanoantennas for visible and infrared radiation. Rep. Prog. Phys. 75, 024402 (2012).

[12] Schuller, J. A., Barnard, E. S., Cai, W. S., Jun, Y. C., White, J. S. \& Brongersma, M. L. Plasmonics for extreme light concentration and manipulation. Nature Mat. 9, 193-204 (2010).

[13] Zijlstra, P., Paulo, P. M. R. \& Orrit, M. Optical detection of single non-absorbing molecules using the surface plasmon resonance of a gold nanorod. Nature Nanotech. 7, 379-382 (2012).

[14] Anger, P., Bharadwaj, P. \& Novotny, L. Enhancement and quenching of single-molecule fluorescence. Phys. Rev. Lett. 96, 113002 (2006).

[15] Kühn, S., Håkanson, U., Rogobete, L. \& Sandoghdar, V. Enhancement of single-molecule fluorescence using a gold nanoparticle as an optical nanoantenna. Phys. Rev. Lett. 97, 017402 (2006).

[16] Kinkhabwala, A., Yu, Z. F., Fan, S. H., Avlasevich, Y., Mullen, K. \& Moerner, W. E. Large single-molecule fluorescence enhancements produced by a bowtie nanoantenna. Nature Photon. 3, 654-657 (2009). 
[17] Aouani, H., Mahboub, O., Bonod, N., Devaux, E., Popov, E., Rigneault, H., Ebbesen, T. W. \& Wenger, J. Bright unidirectional fluorescence emission of molecules in a nanoaperture with plasmonic corrugations. Nano Lett. 11, 637-644 (2011).

[18] Bermúdez Ureña, E., Kreuzer, M. P., Itzhakov, S., Rigneault, H., Quidant, R., Oron, D. \& Wenger, J. Excitation enhancement of a quantum dot coupled to a plasmonic antenna. Adv. Mater. 24, OP314-OP320 (2012).

[19] Busson, M. P., Rolly, B., Stout, B., Bonod, N. \& Bidault, S. Accelerated single photon emission from dye molecule-driven nanoantennas assembled on DNA. Nature Commun. 3, 962 (2012).

[20] Acuna, G. P., Möller, F. M., Holzmeister, P., Beater, S., Lalkens, B. \& Tinnefeld, P. Fluorescence enhancement at docking sites of DNA-directed self-assembled nanoantennas. Science 338, 506-510 (2012).

[21] Choudhury, S. D., Ray, K. \& Lakowicz, J. R. Silver nanostructures for fluorescence correlation spectroscopy: reduced volumes and increased signal i intensities. J. Phys. Chem. Lett. 3, 29152919 (2012).

[22] Lu, G. W., Liu, J. Zhang, T. Y., Li, W. Q., Hou, L., Luo, C. X., Lei, F., Manfait, M. \& Gong, Q. H. Plasmonic near-field in the vicinity of a single gold nanoparticle investigated with fluorescence correlation spectroscopy. Nanoscale 4, 3359-3364 (2012).

[23] Estrada, L. C., Aramendia, P. F. \& Martinez, O. E. 10000 times volume reduction for fluorescence correlation spectroscopy using nano-antennas. Opt. Express 16, 20597-20602 (2008).

[24] Kinkhabwala, A. A., Yu, Z. F., Fan, S. H., \& Moerner, W. E. Fluorescence correlation spectroscopy at high concentrations using gold bowtie nanoantennas. Chem. Phys. 406, 3-8 (2012).

[25] Yuan, H., Khatua, S., Zijlstra, P., Yorulmaz, M. \& Orrit, M. Thousand-fold enhancement of single-molecule fluorescence near a single gold nanorod. Angew. Chem. 125, 1255-1259 (2013).

[26] Rigneault, H., Capoulade, J., Dintinger, J., Wenger, J., Bonod, N., Popov, E., Ebbesen, T. W. \& Lenne, P. F. Enhancement of single-molecule fluorescence detection in subwavelength apertures. Phys. Rev. Lett. 95, 117401 (2005).

\section{Acknowledgments}

The research leading to these results has received funding from the European Commission's Seventh Framework Programme (FP7-ICT-2011-7) under grant agreements 288263 (NanoVista), ERC StG 
278242 (ExtendFRET) and ERC AdG (NanoAntennas), the Spanish Ministry of Science and Innovation and the Agence Nationale de la Recherche under grant ANR-10-INBS-04-01 France Bio Imaging. The authors acknowledge A. Brisson for providing the Annexin sample.

\section{Author contributions}

J.W., H.R., N.F.v.H. and M.G.-P. conceived and designed the experiments; D.P., J.W. and S.B.M. performed the experiments and analyzed the data; M.M. and T.S.v.Z fabricated the antennas; J.W., M.G.-P. and N.F.v.H. wrote the manuscript.

\section{Additional information}

The authors declare no competing financial interests. Supplementary information accompanies this paper at www.nature.com/naturenanotechnology. Reprints and permission information is available online at http://npg.nature.com/reprintsandpermissions/. Correspondence and requests for materials should be addressed to JW. 
a

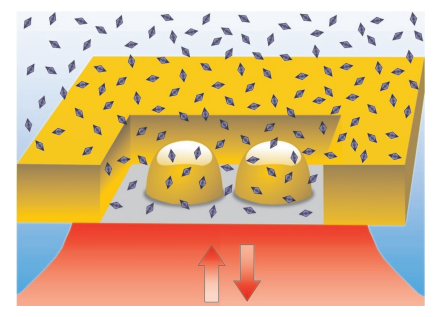

C

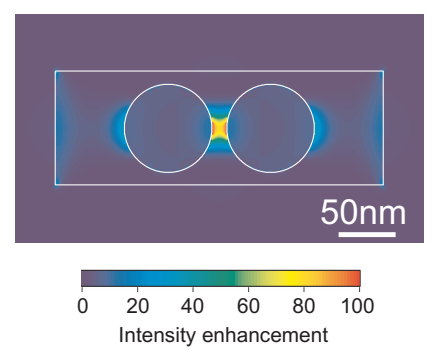

b

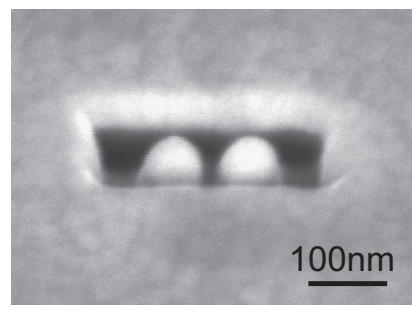

d

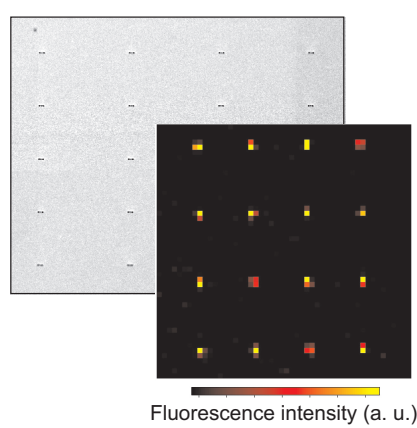

Figure 1: Antenna-in-box platform for single molecule analysis at high concentrations. (a) Schematic of dimer gap antenna inside a rectangular aperture. The device is milled in a gold film (yellow) previously deposited on a glass substrate (gray), and covered by a solution containing fluorescent molecules at micromolar concentration (light blue). Laser illumination (up-pointing arrow) and fluorescence detection (down-pointing arrow) are performed from the glass side. (b) Scanning electron microscopy (SEM) image of a fabricated nanoantenna. (c) Finite-difference time-domain computation of excitation intensity enhancement. The incoming light at a wavelength of $633 \mathrm{~nm}$ is polarized parallel to the dimer axis. (d) SEM and confocal images of an array of nanoantennas with $5 \mu \mathrm{m}$ pitch. 

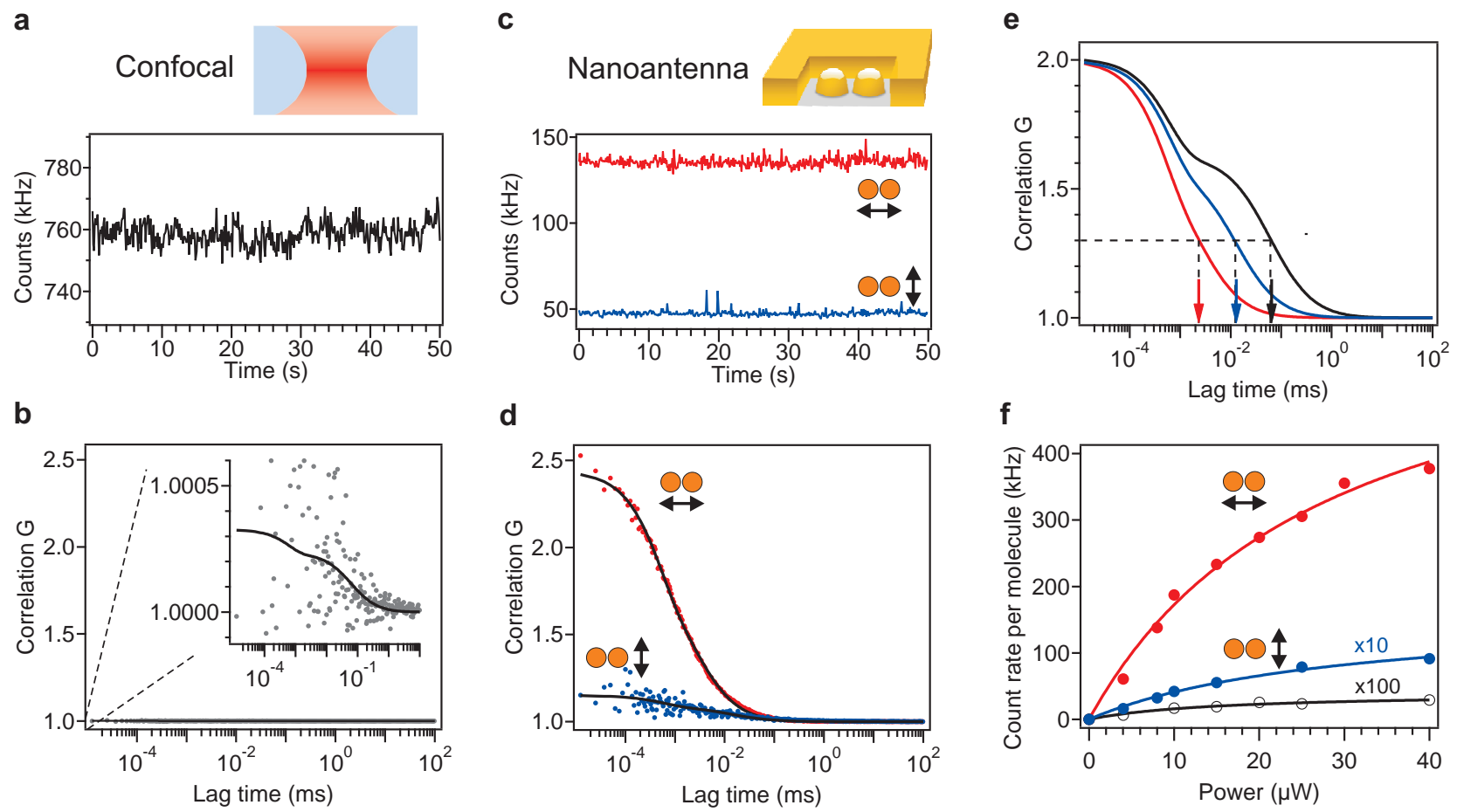

Figure 2: Enhanced single molecule analysis with an antenna-in-box. (a) Fluorescence time trace and (b) FCS correlation function (raw data) for the reference confocal setup. Due to the large number of molecules in the confocal volume, the correlations are almost hidden with amplitudes close to one (the insert provides a close-up view). (c) and (d) display the time traces and correlation functions (raw data) for a nanoantenna with $15 \mathrm{~nm}$ gap size, with the excitation polarisation set parallel (red) or perpendicular (blue) to the nanoantenna. For all data in (a-d), Alexa Fluor 647 concentration is $15.5 \mu \mathrm{M}$, and $200 \mathrm{mM}$ of methyl viologen is used as a chemical quencher. The excitation power is constant at $10 \mu \mathrm{W}$. (e) Normalized FCS curves for the nanoantenna with parallel (red) and perpendicular (blue) polarisation orientation. The reference FCS curve for the diffraction-limited confocal set-up is shown in black. All FCS curves have been scaled vertically to have an amplitude of one unit. Arrows indicate the average residence times for molecules in the observation volumes. The component at short times apparent on the confocal curve is due to fluorescence photoblinking that originates from intersystem crossing and cis-trans photoisomerization of the dye. (f) Fluorescence count rates per molecule versus the excitation power. The data for the nanoantenna with perpendicular polarisation (blue) and the reference confocal set-up (black) are multiplied respectively by 10x and 100x to allow visualization in the same plot. 
a

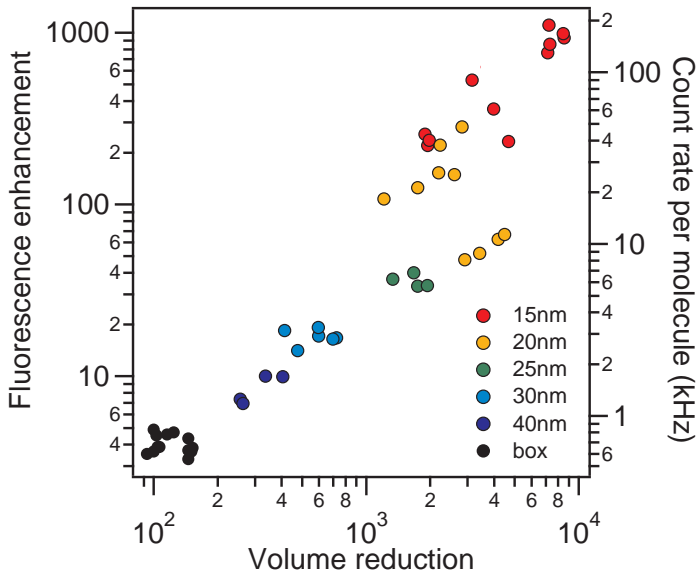

b

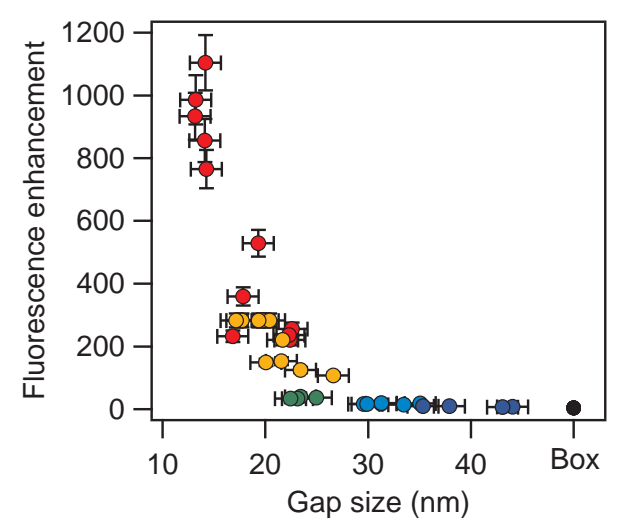

C

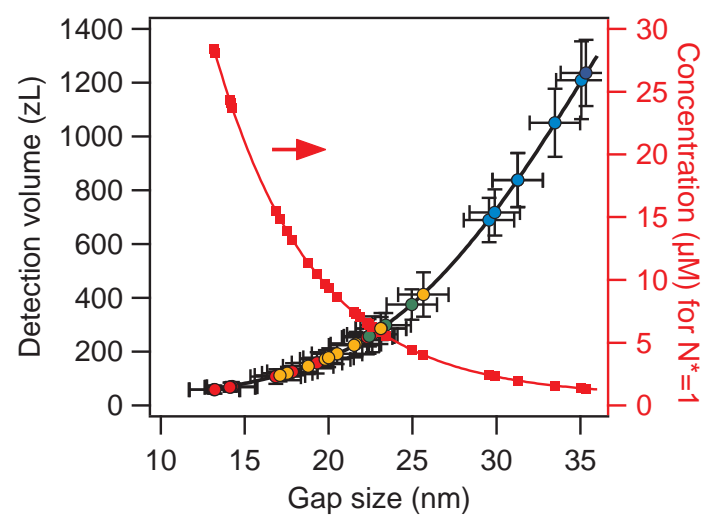

Figure 3: Fluorescence enhancement and volume reduction as function of nanoantenna gap size. (a) Scatter plot of fluorescence enhancement versus volume reduction as compared to the diffraction-limited confocal set-up. The different markers represent different antennas tested, while the color indicates the nominal gap size used for the fabrication. (b) Scatter plot of the fluorescence enhancement factor as a function of gap size calibrated by FCS. (c) Detection volume and concentration for which there is, on average, an individual molecule in the nanoantenna detection volume. Lines are guide to the eyes. Data were empirically found to follow an exponential trend (see Supporting Information Section 8). 

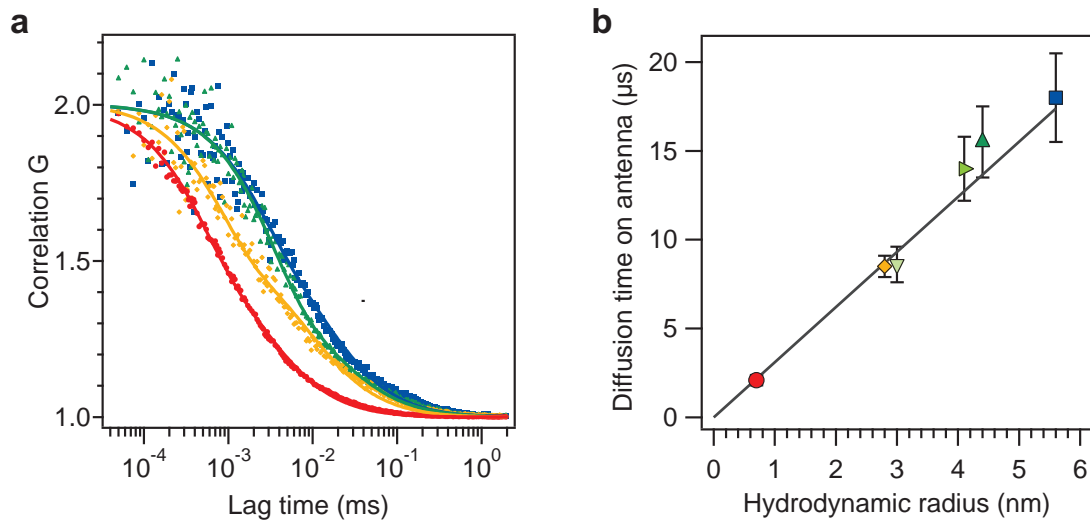

- Alexa647

$\diamond$ Annexin5b - Cy5

$\nabla$ DNA[11bp] - Atto647N (+ Pstl)

$\triangleright$ DNA[40bp] - Atto647N (+ Pstl)

$\triangle$ DNA[51bp] - Atto647N

- ProteinA - Alexa647

Figure 4: Applicability of the antenna-in-box to detect and discriminate individual biomolecules at $10 \mu \mathbf{M}$ concentrations. (a) Normalized fluorescence correlation functions measured on an antenna-in-box of $15 \mathrm{~nm}$ gap size, with excitation polarisation parallel to the antenna axis. The samples are Alexa Fluor 647 free dye (red, molar mass $1.3 \mathrm{kDa}$ ), Annexin 5b (orange, molar mass $36 \mathrm{kDa}$ ), 51 base pairs (bp) double stranded DNA (green, molar mass $32.1 \mathrm{kDa}$ ) and Protein A (blue, molar mass $56 \mathrm{kDa}$ ). The points correspond to experimental data and the solid lines to a numerical fit. (b) Average residence times in the nanoantenna observation volume versus the molecular hydrodynamic radius for the different biomolecules. The black line is the trend expected from Brownian diffusion. 